\title{
Association of Cathepsin B a Salivary Biomarkers in Different Histological Grades of Oral Squamous Cell Carcinoma
}

Alveena Shabbir ( $\sim$ dralveena90@gmail.com )

Dow University of Health Sciences, Pakistan

Humera Waheed

Dow University of Health Sciences, Pakistan

Shaheen Ahmed

Dow University of Health Sciences, Pakistan

Sabhita Shabir Shaikh

Dow University of Health Sciences, Pakistan

\section{Research Article}

Keywords: Oral Squamous Cell Carcinoma, Saliva, ELISA, Cathepsin B, Biomarker, histological grades

Posted Date: November 15th, 2021

DOI: https://doi.org/10.21203/rs.3.rs-1056476/v1

License: (a) (i) This work is licensed under a Creative Commons Attribution 4.0 International License. Read Full License 


\section{Abstract}

\section{Background:}

Oral cancer is considered a major public health problem due to its high mortality and morbidity rates. Survival rate of OSCC can be significantly improved by using non -invasive tool such as salivary biomarkers for detection of OSCC which is considered a promising approach. Cathepsin B is a lysosomal cysteine protein, which is present in abundant quantities in lysosome of cells, tissues and different biological fluids. Increased expression of Cathepsin B is observed in many malignancies including oral cancer. The present study was designed to determine the salivary levels of Cathepsin B in different histological grades of OSCC.

\section{Method:}

In this study total no of 80 research participants were enrolled which were divided into four groups. Each group comprises 20 participants. Groups 1 comprises 20 patients of OSCC (Well differentiated), Group 2 comprises 20 patients of OSCC (moderately differentiated), Group 3 comprises 20 patients of OSCC (poorly differentiated|) and Group 4 comprises 20 healthy controls. Saliva sample was collected from all the four study group and salivary Cathepsin B levels were analyzed by ELISA sandwich technique in duplicate.

\section{Results:}

Salivary levels of Cathepsin B are significantly increased with $p$-value $(<0.001)$ in patients of OSCC in comparison to control group. We observed highest mean Cathepsin B levels in well differentiated OSCC followed by poorly differentiated OSCC and moderately differentiated OSCC.

\section{Conclusion:}

Results of the present study suggests that Cathepsin B has a great value as a salivary biomarker for diagnosis and monitoring of OSCS in different histological grades of OSCC. It could increase the survival rate and further improve the prognosis of OSCC.

\section{Introduction}

Oral cancer is a malignant neoplasm which is considered as a major and alarming health problem of global concern due to increasing prevalence and higher mortality rate ${ }^{1}$. It is the sixth most common and leading cause of mortality worldwide and ranked as the 2 nd most common cancer in Pakistan ${ }^{2,3}$. Oral squamous cell carcinoma (OSCCl) is the most common and frequent type of oral cancer and account for approximately $90 \%$ of all oral cancers ${ }^{4,5}$. Approximate annual incidence rate of oral cancer is 300,000 cases and mortality rate is 145,000 cases worldwide ${ }^{6}$. Most common sites of OSCC occurrence are buccal mucosa, tongue and floor of the mouth ${ }^{7}$. The most important and prevalent risk factors 
responsible for development of OSCC are tobacco, alcohol and smokeless tobacco chewing ${ }^{8}$. The other risk factors include: viral infection HPV16 and HPV18, genetic factors, occupational exposure and nutritional deficiencies such as diet intake of low fruits and vegetables and ultraviolent radiation ${ }^{9}$.The main treatment modalities currently available for OSCC are surgical excision, radiotherapy and chemotherapy ${ }^{10}$.

In spite of the advancement in different treatment regimens, the 5-year survival rate of OSCC is found to be less than $50 \%{ }^{11}$. Although the examination of oral cavity is easily assessable but most OSCC are still diagnosed in an advanced stages ${ }^{12}$. Currently, the gold standard for the diagnosis of OSCC is histopathological examination of a biopsy sample ${ }^{13}$. However, biopsy is an invasive procedure and has various drawbacks such as painful, high cost and fear of cancer spread after performing biopsy, which results in late diagnosis of OSCC ${ }^{14}$. Therefore, there is an urgent need to identify new non- invasive methods which will help us in diagnosis and monitoring of OSCC ${ }^{15}$. Diagnosis and monitoring of OSCC by using salivary biomarkers is considered a promising and ideal tool ${ }^{16}$. Using saliva as a diagnostic tool has several advantages as collection of saliva is simple, safe, non-invasive and cost effective in nature and also it has benefits of ease in sampling, handling and processing ${ }^{17,18}$. Furthermore, due to direct contact between saliva and oral cavity, salivary biomarkers are considered a perfect and classic tool for diagnosis and monitoring of $\mathrm{OSCC}^{19}$. Several salivary biomarkers in oral squamous cell carcinoma has been identified which can be used as diagnostic and prognostic markers in OSCC ${ }^{20}$.

Cathepsin B (CTSB) is a lysosomal cysteine protease and belongs to a family of cysteine cathepsins. It is present in abundant quantities in lysosomes of cells and different biological fluids of body such as saliva and serum ${ }^{21}$. Main physiological functions of Cathepsin B is intracellular degradation of protein and protein turnover ${ }^{22}$. Cathepsin B plays a major and important role in development of cancer and its progression. It is associated with basement membrane dissolution and extracellular matrix degradation, a process which is responsible for tumour progression, growth, metastasis and invasion ${ }^{23}$. Its expression in tissues and serum levels are increased in many types of malignancies including $0 \operatorname{SCC}^{24}$. However, previously none of the studies have reported Cathepsin B levels in saliva in patients of OSCC. Therefore, by analyzing Cathepsin B levels in saliva in different histological grades may benefit diagnosis and monitoring of OSCC by using non-invasive technique. This will further lead to increase survival rate and improved prognosis of the disease.

\section{Materials And Methods}

\section{Study design and setting}

An analytical cross sectional study was conducted during the period of July 2019 to December 2019 after approval from Institutional Review Board of Dow University of Health Sciences (IRB-

1223/DUHS/Approval/2019/34). The recruitment of the study participants of Oral Squamous Cell Carcinoma was carried out from the dental OPD of Dow University of Health Sciences. Recruitment of 
control group participants was carried out from patient's attendant's, friends, family and colleagues. Informed consent in written was obtained from each research participants.

\section{Sample size estimation}

Open Epi online sample size calculator was used for sample size estimation according to the statistics reported by Yang W et $a^{25}$. A parameter of severity of cancer nodal involvement was reported by author 9.53 times increased in patients if Cathepsin B was present compared to patients in which Cathepsin B was absent. A minimum of 80 sample size was calculated (20 in each group) at $80 \%$ power and $95 \%$ confidence interval.

\section{Study groups}

Total no of 80 research participants were enrolled in our study, which were divided into four groups 20 in each group. Group 1 consists of 20 patients of well-differentiated OSCC, Group 2 consists of 20 patients of moderately differentiated OSCC, Group 3 consists of 20 patients of poorly differentiated OSCC and Group 4 consists of healthy controls. Inclusion criteria for OSCC patients includes: Patients of OSCC of both genders, aged 18-65, newly diagnosed and biopsy proven were selected after clinical examination and further confirmed by histopathological examination. OSCC patients were divided into three respective group of OSCC i.e. well, moderate and poorly differentiated according to Broder's histopathological grading criteria. Patients with underlying systemic illness such as rheumatoid arthritis, osteoarthritis, pancreatitis, chronic atrophic gastritis and periodontitis and other malignant tumour such as breast, ovary, lung, gastric and nasopharyngeal were excluded. Patients with a history of smoking and smokeless tobacco products were excluded from the control group. In a pre-designed questionnaire demographic data and type, frequency, duration of chewing habits and site of lesion was obtained for each participants of OSCC.

\section{Saliva collection method}

In a sterilized falcon tube of $15 \mathrm{ml}, 2-5 \mathrm{ml}$ of unstimulated whole saliva was collected from each research participant between 9-11 am. Participants were asked to refrain one hour from eating, chewing and drinking before saliva sample collection. After that, the saliva was immediately taken to laboratory and centrifuged for $15 \mathrm{~min}, 4^{\circ} \mathrm{C}$ at $8000 \mathrm{rpm}$. Saliva supernatant was collected and aliquoted into $1.5 \mathrm{ml}$ of Eppendorf tubes and stored at $-80^{\circ} \mathrm{C}$ until further processing of saliva.

\section{Sample analysis}

Total protein estimation analysis was performed according to Bradford's method ${ }^{26}$. Salivary levels of Cathepsin B in all the four study groups were determined by Enzyme Linked Immunosorbent Assay (ELISA) sandwich technique according to manufacturer's instructions available in the kit (Bioassay Technology KIT: $35054 \mathrm{Hu}$ ). Each sample was analyzed in duplicates for better result outcomes.

\section{Statistical analysis}


Statistical analysis of all the obtained data was analyzed by SPSS version no 21 . For all quantitative variables, mean and standard deviation were recorded and for all categorical variables, percentages and frequencies were recorded. One-way ANOVA test was used for comparison of mean Cathepsin B levels between OSCC groups and control group. Chi square test and Fisher's Exact test was used to determine the association between OSCC groups and control group for patient related factors. For univariate and multivariate analysis linear regression was used as the outcome variable Cathepsin B follows a continuous scale.

\section{Results}

Clinicopathological characteristics of all 80 research participants enrolled in our study are summarized in Table 1. Among the total 60 patients of OSCC, males patient comprises $43(71.66 \%)$ and females patients comprises $17(28.3 \%)$ cases. The male to female ratio observed was 2.5:1. With respect to age factors we observed OSCC patients are distributed evenly in all the three groups of OSCC with the minimum age found was 19 years and maximum age found was 65 years. The most common site of OSCC found in our study in all the three groups of OSCC was buccal mucosa 31 cases $(51.7 \%)$ followed by tongue 10 cases (16.7\%), retromolar area 5 cases (8.3\%), palate and lower buccal sulcus 4 cases each (6.7\%), upper sulcus and lower lip 3 cases each (5\%). With regard to tobacco usage of both forms smoking and smokeless tobacco, we observed that usage of tobacco products for greater than 5 years duration causes increase in no of OSCC cases in all three study groups.

The salivary mean protein levels observed in three groups of OSCC and in control group were as follows: well differentiated OSCC $19.5 \mathrm{mg} / \mathrm{ml}( \pm 14.7)$, moderately differentiated OSCC $27.3 \mathrm{mg} / \mathrm{ml}( \pm 16.2)$ and poorly differentiated OSCC $15.9( \pm 9.9) \mathrm{mg} / \mathrm{ml}$ and in control group $3.4 \mathrm{mg} / \mathrm{ml}( \pm 1.9)$ as shown in Table 2 and Figure 1. Statistically significant difference with $p$-value $(<0.001)$ was observed in salivary mean protein levels between OSCC and control group. Highest protein concentration was observed in moderately differentiated OSCC followed by well differentiated OSCC and poorly differentiated OSCC. Lowest salivary mean protein levels were observed in control group.

Among the total 60 OSCC patients, Cathepsin B in saliva was detected in $45(70 \%)$ cases where as in the control group salivary Cathepsin B was detected in only $3(15 \%)$ cases. The salivary mean Cathepsin B levels observed in three groups of OSCC were as follows: well differentiated OSCC patients $3.7( \pm 1.8)$ $\mathrm{ng} / \mathrm{ml}$, moderately differentiated OSCC patients $1.0( \pm 1.3) \mathrm{ng} / \mathrm{ml}$ and poorly differentiated OSCC patients was $3.3( \pm 3.5) \mathrm{ng} / \mathrm{ml}$. Statistically significant difference with $\mathrm{p}$-value $(<0.001)$ was observed in salivary mean Cathepsin B levels between OSCC and control group. Highest salivary Cathepsin B levels were observed in well differentiated OSCC followed by poorly differentiated OSCC and moderately differentiated OSCC as shown in Table 3, Figure 2.

The association of Cathepsin B levels was only found within the four study groups, OSCC and control group. No significant association of salivary Cathepsin B levels with age, gender, site, frequency and duration of tobacco intake was observed as shown in Table 5. 
Univariate and multivariate analyses was performed to observe the association of salivary Cathepsin B levels with clinicopathological characteristics and study groups. The only variable, which observed significant association with Cathepsin B levels, was the OSCC/non-OSCC group. Therefore, multivariate analysis execution was not possible. Interpretations of regression (B) coefficients were as follows: for well-differentiated OSCC patients $3.37 \mathrm{ng} / \mathrm{ml}$, for moderately differentiated OSCC patients $0.63 \mathrm{ng} / \mathrm{ml}$ and for poorly differentiated OSCC patients $2.99 \mathrm{ng} / \mathrm{ml}$ estimated increase in Cathepsin B levels was observed respectively as shown in Table 6.

\section{Discussion}

Oral squamous cell carcinoma worldwide is one of the most common malignant tumour, which has high rates of morbidity and mortality ${ }^{27}$. The main reason behind this is that most OSCC when diagnosed are in advanced stages ${ }^{28}$. The diagnosis and monitoring of OSCC by using salivary biomarkers is considered a promising approach for lowering oral cancer burden and increases the overall survival rate ${ }^{29}$. This is the first study in which we have analyzed salivary levels of Cathepsin B in different histological grades of oscC.

Cathepsin B role in cancer is attributed to extracellular matrix degradation resulting in tumour invasion and metastasis ${ }^{30}$. Cathepsin $\mathrm{B}$ is considered a promising biomarker as increased expression of Cathepsin B is reported in various types of metastasis and invasive cancers such as lung, breast, ovary, colon, gastric, nasopharyngeal and oral cancer ${ }^{23}$. Lihua Xu et al., in his research has reported increased expression of Cathepsin B in gastric carcinoma as compared to normal tissue which is found to be significantly associated with tumor size $(P<0.001)$, Tumour Node Metastasis $(T N M)$ Stage $(P<0.001)$ and decreased overall survival $(P<0.001)^{31}$. In malignant ovarian cancers, Cathepsin $B$ levels are significantly elevated in cystic fluid as reported by Kolwijck et al., ${ }^{32}$. Chan et al., in his research studied Cathepsin B in 588 colon cancer patients and found that $82 \%$ patients were positive for Cathepsin B which was further confirmed by Cavallo-Medved et al., and he concluded that elevated levels of Cathepsin $B$ are associated with poor prognosis ${ }^{33}$. In nasoparyngeal carcinoma Cathepsin B levels are significantly elevated in serum as compared to healthy controls which was significantly associated with TNM $(p=$ 0.001 ) as observed by Gongjun Tan et al., ${ }^{24}$. In breast cancer patients Schraufstatter et al., observed increases Cathepsin $B$ in serum with $p$-value $(p=0.015)$ as compared to normal control ${ }^{32}$.

In our study, we observed OSCC patients are evenly distributed in all age groups. Prevalence of OSCC is high in male patients as compared to female patients. Buccal mucosa as the most common site of OSCC occurrence is observed in our study. We also observed tobacco consumption of both forms smoking and smokeless tobacco for greater than 5 years duration causes increase in no of OSCC cases.

In our study, Cathepsin B was observed in $45(70 \%)$ patients out of total 60 OSCC patients and in control group Cathepsin was detected in only $3(15 \%)$ of research participants. Mean salivary total protein levels are increased significantly with p-value $(<0.001)$ in patients of OSCC as compared to control group. Kavya 
Prabhu M Sayeeda Mussavira et al., and Hivashankara AR has also reported in there studies that salivary protein levels are increased significantly in OSCC patients in comparison to control group ${ }^{34,35}$. Proteins are accountable for most functions of saliva such as physical protection, lubrication, buffering, tooth integrity maintenance and antibacterial activity ${ }^{36}$. Total salivary proteins are increased in patients of OSCC probably due to the ongoing inflammatory response, which triggers the sympathetic activity and increases the synthesis and production of some proteins to increase the shielding effect of saliva and provide protective function to combat against $\mathrm{OSCC}^{36}$. Furthermore, increase in salivary total proteins occurs to combat the violation and aberration in capillaries and mucosal lining as a result of inflammation in $\mathrm{OSCC}^{37}$. In our study, we observed highest total salivary protein levels in moderately differentiated OSCC group followed by well differentiated and poorly differentiated group of OSCC. This could be due to the fact that total salivary protein levels in patients of OSCC is dependent on several factors relatable to patients such as (diet, gender and age) and factors related to disease such as (infection, metastasis and lymph node invasion) ${ }^{38}$.

We determined the levels of salivary Cathepsin B in OSCC patients in different histological grades. It was observed that salivary levels of Cathepsin B are increased significantly with p-value $(<0.001)$ in patients of OSCC as compared to control group. Increased expression of Cathepsin B in tissues, serum and cell line are reported by several researches. Wei-En Yang et al, in his research has observed increased CTSB expression in patients of OSCC which was associated with higher tumour grade $(p=0.008)$ and lymph node metastasis $(p=0.007)^{25}$. X. Yang et al., has reported increased CTSB protein and mRNA levels in cell lines and tissues of OSCC patients as compared to adjacent non -malignant tissues by Polymerase Chain Reaction (PCR), western blotting and immunohistochemistry analysis ${ }^{39}$. Yousuf Saleh et al., in tongue cancer patients has observed elevated Cathepsin B in serum and tumor tissues associated with high tumour grade ${ }^{40}$.

The highest mean Cathepsin B levels in well differentiated OSCC group followed by poorly differentiated OSCC group, moderately differentiated OSCC group and controls were observed in our study. The variable results observed in our study could be due to the fact that Broder's histopathological grading of OSCC provided us information only about degree of differentiation of cell which is only one parameter ${ }^{41}$. However, prognosis of OSCC apart from histological grading also depends on other factors such as tumour size and lymph node invasion and metastasis ${ }^{42}$. Furthermore, as Cathepsin B is an inflammatory marker and more inflammatory cells are present in initial grades of OSCC that's why may be the plausible reason we observed increased Cathepsin B salivary levels in well differentiated OSCC ${ }^{43}$. Smitha Kullage et al., in his research has also reported high inflammatory cell count in patients of well differentiated OSCC in correspondence to moderate and poorly differentiated groups of OSCC ${ }^{44}$. Cathepsin B role in cancer is related to basement membrane and extracellular matrix protein degradation, a mechanism which is responsible for metastasis and tumour invasion of cancer cells. It activates and initiates a proteolytic cascade in which urokinase plasminogen activator, matrix metalloproteinase and plasminogen all together causes degradation of extracellular matrix components. Cathepsin B also causes degradation of cell adhesion protein E cadherin at adherin junctions, which causes detachment of 
cells. This altogether results in cell invasion, progression of tumour and metastases ${ }^{45,46}$. That's may be the plausible reason we observed increased salivary Cathepsin B levels in poorly differentiated OSCC.

\section{Conclusion}

In our study, salivary Cathepsin B levels are significantly increased in patients of OSCC as compared to healthy controls. Thus, Cathepsin B can be considered a helpful and beneficial salivary biomarker for diagnosis and monitoring of OSCC in different histological grades. Timely detection and treatment will increase the survival rate of OSCC and further reduce the mortality and morbidity rate. The results of the present study makes the way for future studies on Cathepsin B as a non- invasive tool salivary biomarker for diagnosis and monitoring of OSCC.

\section{Abbreviations}

OSCC

Oral Squamous Cell Carcinoma

CTSB

Cathepsin B

ELISA

Enzyme Linked Immunosorbent Assay

TNM

Tumor Node Metastasis

PCR

Polymerase Chain Reaction.

\section{Declarations}

\section{Acknowledgments}

We are very grateful and like to thank to all the research participants for their cooperation and giving precious time and consent for participating in our study.

\section{Authors contribution}

AS: contributed in conceptualizing the overall study, data collection, sample processing and drafting of manuscript. H.W. and S.H supervised the study and contributed in conceptualizing the overall study, data collection, sample processing, drafting and editing of manuscript S.S contributed in data collection and helped in assessments of patients of Oral Squamous Cell Carcinoma.

\section{Funding}

Self-funded research, no funding for the current study was received by any organization. 
Availability of data and materials

All data used, generated and analyzed in this current study is available on reasonable request from authors.

\section{Ethics approval and consent to participate}

The present study is completed after approval from Institutional Review Board of Dow University of Health Sciences IRB Number: IRB-1223/DUHS/Approval/2019/34. Informed consent in written from all the research participants was obtained.

\section{Consent for publication}

The current study does not contain any images or videos related to any research participant. The consent for publication is obtained from all research participants included in the current study.

\section{Competing interests}

The authors declare that they have no conflict of interest.

\section{Authors detail}

1. Department of Oral Medicine, Dow University of Health Sciences from Pakistan (Corresponding author)

2. Department of Dow College of Biotechnology, Dow University of Health Sciences from Pakistan,

3. Department of Oral and Maxillofacial Surgery, Dow University of Health Sciences from Pakistan

4. Department of NILGID, Dow University of Health Sciences from Pakistan.

\section{References}

1. Chaves FN, Bezerra TMM, Moraes DC, dos Santos Costa SF, Silva PGB, Alves APNN, et al. Loss of heterozygosity and immunoexpression of PTEN in oral epithelial dysplasia and squamous cell carcinoma. Exp Mol Pathol. 2020;112:104341.

2. Sahu S, Routray S. Assessing the analytical efficacy of TEX in diagnosing oral cancer using a systematic review approach. J Oral Pathol Med. 2021;50(2):123-28.

3. Khan Z, Dreger S, Shah SMH, Pohlabeln H, Khan S, Ullah Z, et al. Oral cancer via the bargain bin: the risk of oral cancer associated with a smokeless tobacco product (Naswar). PloS One. 2017; 12(7):e0180445.

4. Gaba Fl, Sheth CC, Veses V. Salivary biomarkers and their efficacies as diagnostic tools for oral squamous cell carcinoma: systematic review and meta-analysis. J Oral Pathol Med. 2021;50(3):299-307. 
5. Principe S, Zapater-Latorre E, Arribas L, Garcia-Miragall E, Bagan J. Salivary IL-8 as a putative predictive biomarker of radiotherapy response in head and neck cancer patients. Clin Oral Investig. 2021:1-12.

6. Pérot P, Falguieres M, Arowas L, Laude H, Foy J-P, Goudot P, et al. Investigation of viral etiology in potentially malignant disorders and oral squamous cell carcinomas in non-smoking, non-drinking patients. PloS One. 2020;15(4):e0232138.

7. Pałasz P, Adamski Ł, Górska-Chrząstek M, Starzyńska A, Studniarek M. Contemporary diagnostic imaging of oral squamous cell carcinoma-a review of literature. Pol J Radiol. 2017;82:193.

8. Chen Q, Shao Z, Liu K, Zhou X, Wang L, Jiang E, et al. Salivary Porphyromonas gingivalis predicts outcome in oral squamous cell carcinomas: a cohort study. BMC Oral Health. 2021;21(1):1-9.

9. Dikova V, Jantus-Lewintre E, Bagan J. Potential non-invasive biomarkers for early diagnosis of oral squamous cell carcinoma. J Clin Med. 2021;10(8):1658.

10. de Barros-Silva PG, Fontes-Borges MM, Costa-Dias C, Mota-Lemos JV, do Perpétuo SocorroSaldanha-Cunha M, Fernandes-Souza E, et al. Clinical-pathological and sociodemographic factors associated with the distant metastasis and overall survival of oral cavity and oropharynx squamous cell carcinoma. Med Oral Patol Oral Cir Bucal. 2020;25(3):e375.

11. Bjerkli I-H, Jetlund O, Karevold G, Karlsdóttir Á, Jaatun E, Uhlin-Hansen L, et al. Characteristics and prognosis of primary treatment-naive oral cavity squamous cell carcinoma in Norway, a descriptive retrospective study. PloS One. 2020;15(1):e0227738.

12. Cristaldi M, Mauceri R, Di Fede O, Giuliana G, Campisi G, Panzarella V. Salivary biomarkers for oral squamous cell carcinoma diagnosis and follow-up: current status and perspectives. Front. physiol. 2019;10:1476.

13. Cui Y, Kim H-S, Cho ES, Han D, Park JA, Park JY, et al. Longitudinal detection of somatic mutations in saliva and plasma for the surveillance of oral squamous cell carcinomas. PloS one. 2021;16(9):e0256979.

14. Nakamichi E, Sakakura H, Mii S, Yamamoto N, Hibi H, Asai M, et al. Detection of serum/salivary exosomal Alix in patients with oral squamous cell carcinoma. Oral Dis. 2021;27(3):439-47.

15. Liu D, Xin Z, Guo S, Li S, Cheng J, Jiang H. Blood and salivary micrornas for diagnosis of oral squamous cell carcinoma: a systematic review and meta-analysis. J Oral Maxillofac Surg. 2020. 79(5):1082.e1-1082.e13.

16. Naz S, Salah K, Khurshid A, Hashmi AA, Faridi N. Head and neck squamous cell carcinomacomparative evaluation of pathological parameters in young and old patients. Asian Pac J Cancer Prev. 2015;16(9):4061-63.

17. Al Rawi N, Almabrok N, Kou RA, Mkadmi S, Rizvi Z, Hamdoon Z. The role of differentially expressed salivary microRNA in oral squamous cell carcinoma. a systematic review. Arch Oral Biol. 2021:105108.

18. Diesch T, Filippi C, Fritschi N, Filippi A, Ritz N. Cytokines in saliva as biomarkers of oral and systemic oncological or infectious diseases: a systematic review. Cytokine. 2021:155506. 
19. Chiamulera MMA, Zancan CB, Remor AP, Cordeiro MF, Gleber-Netto FO, Baptistella AR. Salivary cytokines as biomarkers of oral cancer: a systematic review and meta-analysis. BMC cancer. 2021;21(1):1-16.

20. Kaur J, Jacobs R, Huang Y, Salvo N, Politis C. Salivary biomarkers for oral cancer and pre-cancer screening: a review. Clin Oral Investig. 2018;22(2):633-40.

21. Ahmed MAA, Mohammed-Salih HS, Yassir YA, Al-Judy HJ. Time-related salivary cathepsin B levels and periodontal status in different orthodontic force magnitudes. J Baghdad Coll Dent. 2015;27(2):115-22.

22. Kos J, Mitrović A, Mirković BJ. The current stage of cathepsin B inhibitors as potential anticancer agents. Future Med Chem. 2014;6(11):1355-71.

23. Gondi CS, Rao JS. Cathepsin B as a cancer target. Expert Opin Ther Targets. 2013;17(3):281-91.

24. Tan G, Liu Q, Tang X, Kang T, Li Y, Lu J, et al. Diagnostic values of serum cathepsin B and D in patients with nasopharyngeal carcinoma. BMC cancer. 2016;16(1):1-7.

25. Yang W-E, Ho C-C, Yang S-F, Lin S-H, Yeh K-T, Lin C-W, et al. Cathepsin B expression and the correlation with clinical aspects of oral squamous cell carcinoma. PLoS One. 2016;11(3):e0152165.

26. Bradford MM. A rapid and sensitive method for the quantitation of microgram quantities of protein utilizing the principle of protein-dye binding. Anal Biochem. 1976;72(1-2):248-54.

27. Ankita K, Shwetha V, Vanitha S, Sujatha SR, Nagaraju R, Pavan KT. Assessment of salivary endothelin-1 in patients with leukoplakia, submucous fibrosis, oral cancer and healthy individuals-a comparative study. J Stomatol Ora/ Maxillofac Surg. 2019;120(4):326-31.

28. Smriti K, Ray M, Chatterjee T, Shenoy R-P, Gadicherla S, Pentapati K-C, et al. Salivary MMP-9 as a biomarker for the diagnosis of oral potentially malignant disorders and oral squamous cell carcinoma. Asian Pac J Cancer Prev. 2020;21(1):233.

29. He L, Ping F, Fan Z, Zhang C, Deng M, Cheng B, et al. Salivary exosomal miR-24-3p serves as a potential detective biomarker for oral squamous cell carcinoma screening. Biomed Pharmacother. 2020;121:109553..

30. Gondi CS, Rao JS. Cathepsin B as a cancer target. Expert Opin Ther Targets. 2013;17(3):281-91.

31. Xu L, Peng S, Zhang N, Liu R, Huang Q, Li X, et al. Expression status of cathepsin B may as a prognostic marker for human gastric carcinoma. Int J Clin Exp Pathol. 2016;9(7):6988-98.

32. Ruan H, Hao S, Young P, Zhang H. Targeting cathepsin B for cancer therapies. Horizons in cancer research. 2015;56:23.

33. Chen S, Dong H, Yang S, Guo H. Cathepsins in digestive cancers. Oncotarget. 2017;8(25):41690.

34. Mussavira S, Kumar HH, Bindhu O. Evaluation of sialic acid, uric acid, total protein and amylase activity in biofluids of OSCC patients. Res J Life Sci. 2020.

35. Shivashankara A, Kavya Prabhu M. Salivary total protein, sialic acid, lipid peroxidation and glutathione in oral squamous cell carcinoma. Biomed Res. 2011;22(3). 
36. Kumar JS, Gayathri R, Priya VV. Evaluation of salivary total proteins, albumin, globulin, and albumin/globulin ratio among healthy individuals and patients with chronic gingivitis. Drug Invent Today. 2018;10(6).

37. Hasan HR, Abdelwahb NN. Evaluation of protein level's changes in saliva and sera of oral squamous cell carcinoma patients. Baghdad Sci. J. 2014;11(2).

38. Al-Muhtaseb SI. Serum and saliva protein levels in females with breast cancer. Oncol Lett 2014;8(6):2752-56.

39. Yang X, Wei K-j, Zhang L, Pan H-y, Li J, Zhong L-p, et al. Increased expression of Cathepsin B in oral squamous cell carcinoma. Int J Oral Maxillofac. 2010;39(2):174-81.

40. Saleh Y, Wnukiewicz J, Trziszka T, Siewinski M, Ziolkowski P, Kopec W. Cathepsin B and cysteine protease inhibitors in human tongue cancer: Correlation with tumor staging and in vitro inhibition of cathepsin B by chicken cystatin. J Cancer Mol. 2006;2:67-72.

41. Akhter M, Hossain S, Rahman QB, Molla MR. A study on histological grading of oral squamous cell carcinoma and its co-relationship with regional metastasis. J Oral Maxillofac Pathol. 2011;15(2):168.

42. Jayade BV, Bhat K, Patil B, Nayak R, Sant A. Histological significance of p53 gene expression in squamous cell carcinoma of the buccal mucosa. J Ora/ Maxillofac Surg. 2009;8(3):205.

43. Fonović M, Turk B. Cysteine cathepsins and extracellular matrix degradation. Biochim Biophys Acta. 2014;1840(8):2560-70.

44. Kullage S, Jose M, Shanbhag VKL, Abdulla R. Qualitative analysis of connective tissue stroma in different grades of oral squamous cell carcinoma: a histochemical study. Indian J Dent Res. 2017;28(4):355.

45. Lampe C, Gondi C. Cathepsin B inhibitors for targeted cancer therapy. J Cancer Sci Ther. 2014;6(10):417-21.

46. Turk V, Stoka V, Vasiljeva O, Renko M, Sun T, Turk B, et al. Cysteine cathepsins: from structure, function and regulation to new frontiers. Biochim Biophys Acta. 2012;1824(1):68-88.

\section{Tables}

Due to technical limitations, table 1 to 6 is only available as a download in the Supplemental Files section.

\section{Figures}




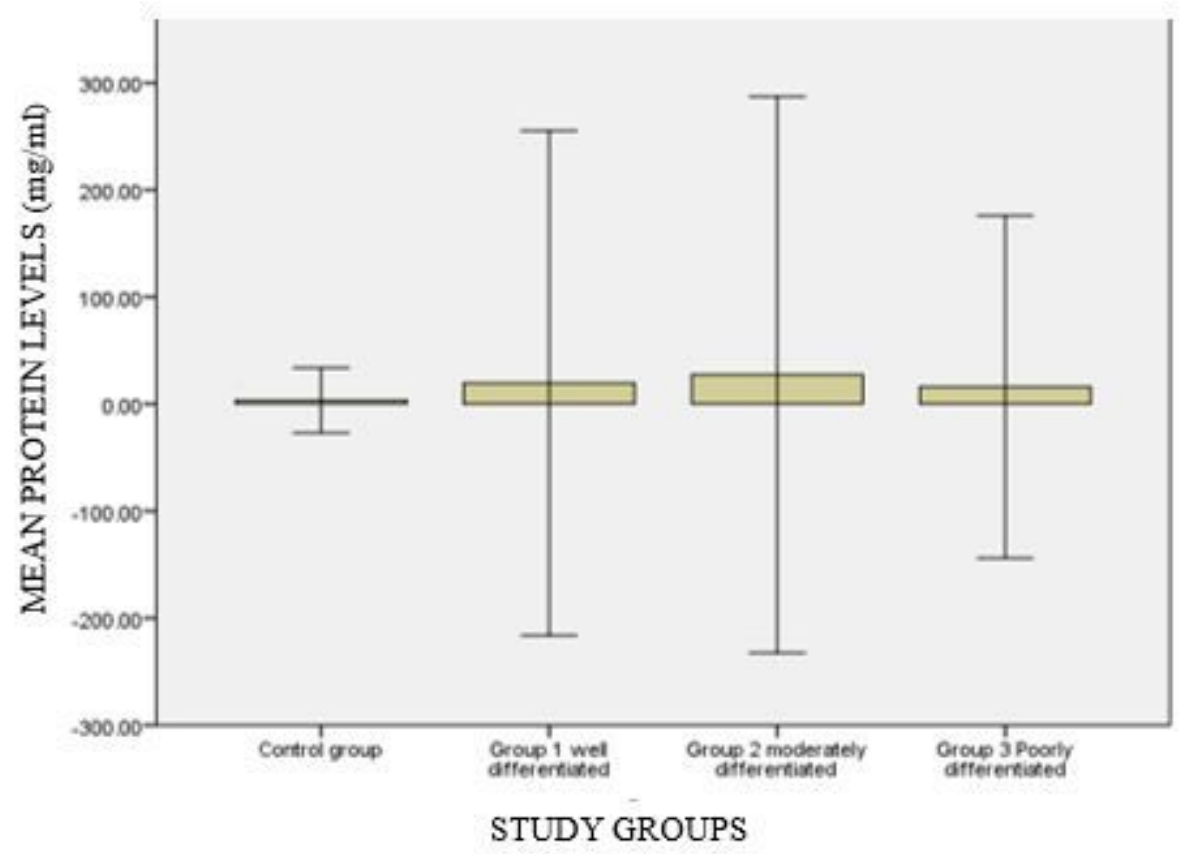

Figure 1

Box plot showing mean salivary protein levels of OSCC and control group

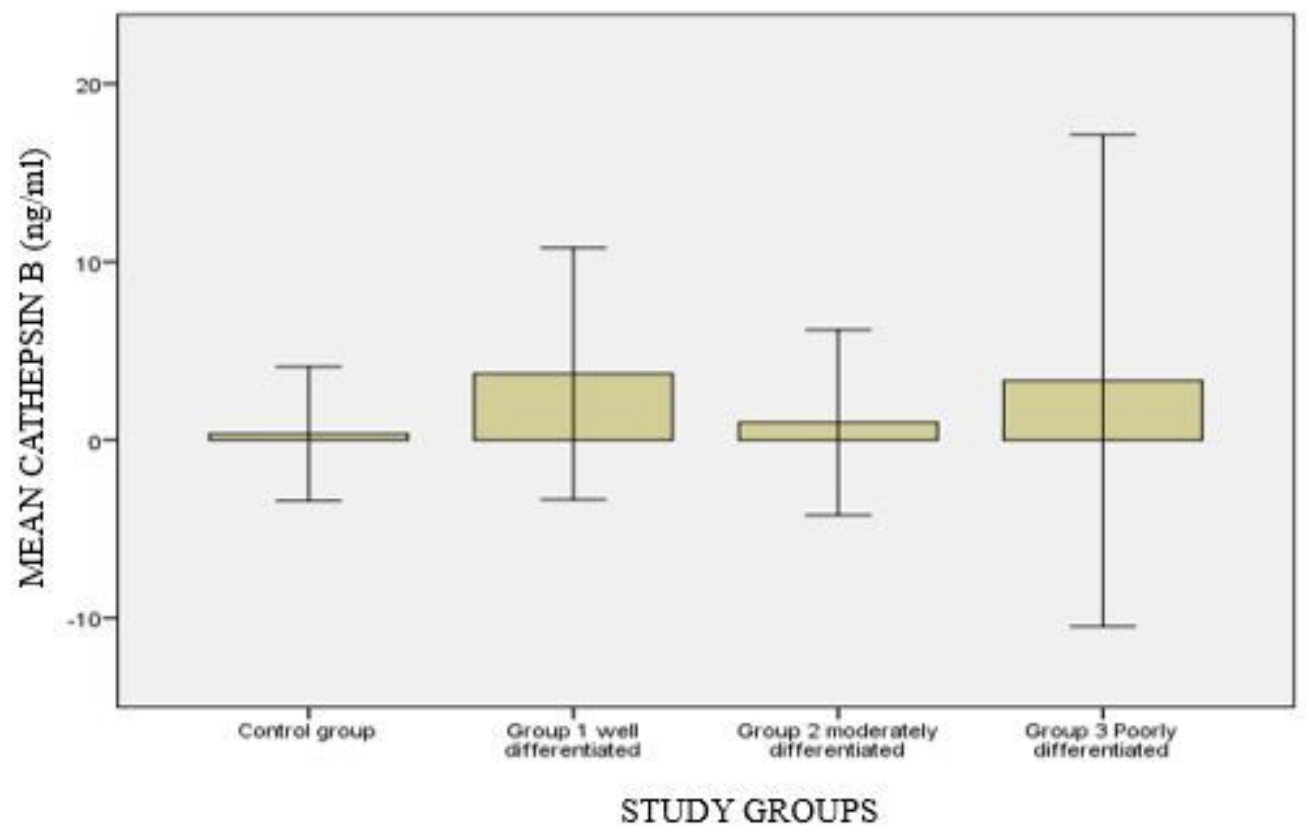

Figure 2

Box plot of mean salivary cathepsin B levels of OSCC and control group.

\section{Supplementary Files}

This is a list of supplementary files associated with this preprint. Click to download. 
- floatimage1.jpeg

- floatimage2.jpeg

- floatimage4.jpeg

- floatimage6.jpeg

- floatimage7.jpeg

- floatimage8.jpeg 